\title{
Limitations of diagnostic radiology for frail and vulnerable elderly cancer patients
}

\author{
"Over the past 20 years, several outcome studies on the treatment of elderly \\ cancer patients have led to an important progression in the assessment of the \\ elderly cancer patient and, therefore, to an increased treatment tolerance."
}

Owing to longer life expectancy, the incidence of cancer in the elderly and its resulting burden are increasing [1]. Currently, more than two-thirds of deaths from cancer in Europe and in the USA occur in individuals over the age of 65 years, and approximately 60 and $40 \%$ of new cancer cases occur in individuals older than 65 and 70 years, respectively. Moreover, the size of this problem is also increasing in less developed countries. A large number of retrospective studies and prospective clinical trials conducted during the last decade considerably deepened our knowledge of the various solid tumors affecting elderly patients. Over the past 20 years, several outcome studies on the treatment of elderly cancer patients have led to an important progression in the assessment of the elderly cancer patient and, therefore, to an increased treatment tolerance. One of the factors limiting the use of standard chemotherapy regimens in elderly cancer patients is the associated toxicity. As a result, medical oncologists need to carefully consider the information provided by the Multidimensional Geriatric Evaluation before administering chemotherapy. In order to identify the optimal therapeutic approach, it is essential to integrate several factors, such as comorbidities, functional status, depression and mental impairment. Among older patients, a distinction can be made between those in good general health who can be treated as adult patients, frail patients who are mostly treated with palliative care and patients who are in an intermediate state - that is, vulnerable patients who can be treated with adjusted or reduced chemotherapy regimens $[2,3]$.

Even in the field of surgery in elderly cancer patients, good progress has been made. As an example, in order to predict the surgical risk of elderly patients, the current surgical and geriatric assessment tools have been combined to obtain a comprehensive clinical evaluation, in terms of health and functionality. This set of tools now forms the 'Preoperative Assessment of Cancer in the Elderly' and represents a useful means of assessing the suitability for surgery of geriatric cancer patients.

Furthermore, the possible limitations of administering of radiotherapy to the elderly patient have also been studied [4]. While extensive knowledge of the therapeutic approaches for these patients is currently available, so far, little attention has been paid to one of the key aspects of the diagnostic approach - that is, diagnostic radiology. When dealing with elderly cancer patients, there can be many limitations in this field. To the best of our knowledge, no data are available on this subject.

\section{Call for attention to diagnostic radiology for elderly cancer patients}

This topic might require some consideration on the most relevant associated issues, which appear to be worthy of in-depth analysis through the publication of dedicated papers.

In general, according to the division conceived by geriatric oncologists, patients aged 70 years and over can be divided into three categories: patients in good general health, vulnerable patients and frail patients. This division represents a starting point in the assessment of elderly patients. Based on this observation, one might assume that the diagnostic management of elderly patients in good general health should not differ from that which applies to adult patients. On the contrary, the diagnostic approach to vulnerable and frail elderly patients may be different. Thus far, the percentages of frail and vulnerable cancer patients undergoing diagnostic radiological examination who develop associated complications are unknown. The aforementioned complications may arise from possible difficulties in collecting clinical information and written informed consent before performing diagnostic procedures, as well as from clinical contraindications, logistical problems and inadequate communication with patients.
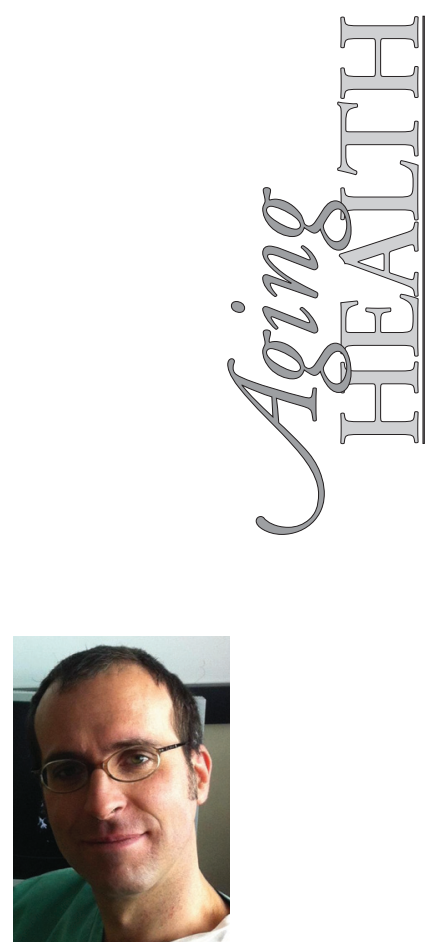

Lorenzo Monfardini Author for correspondence: Interventional Radiology Unit, European Institute of Oncology, Milan, Italy

lorenzo.monfardini@ieo.it

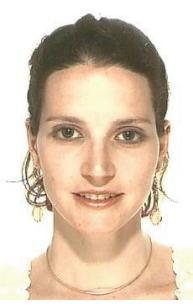

Vittoria Vecchi

School of Medicine, Università degli Studi di Milano, Milan, Italy 


\section{Communication \& written informed consent}

Clinical information plays a key role in diagnostic radiology; the knowledge of a clinical problem, possibly also explained by the patient, may justify a shift from a radiological examination to a different, more appropriate one. However, obtaining medical information from elderly patients can be very difficult. Elderly patients may be mentally impaired or affected by dementias, depression or have no family support.

As for the written informed consent to perform diagnostic procedures, the same issues may be applicable. In spite of being a worldwide well-defined procedure, the written informed consent of frail elderly patients may be difficult or even impossible to obtain, due to the abovementioned age-related conditions.

\section{Clinical contraindications}

With regard to possible clinical contraindications, the performance of a chest $\mathrm{x}$-ray in the case of a primary or metastatic lung neoplasm does not seem to have any limitations.

It is worth mentioning that a CT scan, a primary tool in many clinical conditions, when performed without intravenous administration of contrast media, provides useful information relating solely to lung parenchyma. In order to administer iodinated contrast media, the patient's value of serum creatinine must be in the normal range. Due to the age-related decline in renal function, creatinine clearance tends to decrease in the elderly [5]. For this reason, the possibility of performing CT scans with contrast media in elderly patients is limited.

"...we would like to emphasize the need for a thorough analysis of various epidemiological and clinical aspects of managing a growing population of elderly cancer patients."

Moreover, diabetes, which affects $20 \%$ of people over 75 years of age [6], represents a contraindication to the administration of contrast media when patients are treated with oral antidiabetics. However, the actual percentage of patients affected by this disease who are excluded from the possibility of having CT scans with the use of contrast media is not known.

While CT scans can be performed even if the patient is unable to maintain a fixed position, the execution of an nuclear magnetic resonance requires the avoidance of any movement. This requirement represents a certain limitation for patients affected by Parkinson's disease or by dementia. Once again, however, no data on the feasibility of nuclear magnetic resonance in frail elderly cancer patients are currently available.

Finally, we cannot see clinical contraindications to ultrasound examinations.

\section{Accessibility}

Frail and vulnerable elderly patients are frequently hospitalized at the first diagnosis of cancer. Once hospitalized, they can easily undergo radiological examinations. However, after being discharged from hospital, these patients need to be assisted at home or in nursing homes. With regards to frail patients who need to undergo radiological examinations and are unable to use public transport, logistical difficulties and associated costs may be relevant.

\section{“Too often, diagnostic examinations are chosen according to social and family resources, rather than for clinical reasons."}

Moreover, the reduced ability of the elderly to dress and undress before and after the examination may extend the time required to perform it.

In general, these abovementioned problems are rarely considered while planning radiological follow-up for these patients.

Finally, a proper doctor-patient relationship is based on a mutual understanding that often implies the need for a personal contact with the radiologist, which is not always possible for the patient, for the same logistical reasons.

\section{Screening mammography}

Screening and diagnostic mammography has been thoroughly investigated. A wealth of information in this field is currently available. Surveys carried out by the Surveillance Epidemiology and End Results program indicate that the rate of death from breast cancer in elderly women correlates with the stage of disease at first diagnosis and with the number of coexisting diseases at that time. However, no solid data on screening mammography in women older than 70 years are available. In elderly women, the performance of regular screening mammography can be hampered by several limitations, which may originate from the patient herself or from her primary care physician, as well as from the healthcare system.

It has been demonstrated that patient vulnerability/frailty and the presence of comorbidities are associated with delayed diagnosis of breast cancer. Late detection of breast cancer resulting 
from breast self-examination occurs more frequently than early detection from mammograms [7]. Screening in women aged 70-75 years may be suitably planned according to an individual patient decision, based on risks and benefits, personal preferences, physiological age and life expectancy [8]. Not only the age of the patients, but a complete Multidimensional Geriatric Evaluation, should guide the decision to target elderly women to the screening mammography.

\section{Are diagnostic examinations in elderly patients worthwhile?}

The presence of severe comorbidities in elderly patients corresponds to a reduction of life expectancy, which may limit the performance of radiologic examinations. Although this may seem to be an obvious consideration, this topic is a matter of discussion, since no guidelines are available. The decision to perform diagnostic radiological examinations should be based on the possibility of cancer treatment: when prospected and performed, a diagnostic evaluation is essential.

In this regard, it is worth mentioning the case of elderly bedridden frail patients at risk of excessive toxicity induced by chemotherapy, whose neoplastic lesions need to be reassessed after chemotherapy through diagnostic radiologic examinations.

\section{Conclusion}

Little attention has been paid to the problems associated with the practice of diagnostic radiology in elderly cancer patients. No data are available on the percentage of vulnerable and frail elderly cancer patients undergoing diagnostic radiological examinations, or on their exclusion due to the abovementioned age-related conditions.

Frequently, elderly patients undergo radiological examinations selected by general practitioners, medical oncologists, geriatricians and their relatives, prior to a proper discussion with the radiologists. Too often, diagnostic examinations are chosen according to social and family resources, rather than for clinical reasons.

In order to perform a radiological assessment of these patients, the results of a Multidimensional Geriatric Evaluation may be helpful to clinical radiologists and should be carefully considered.

In this brief editorial, we would like to emphasize the need for a thorough analysis of various epidemiological and clinical aspects of managing a growing population of elderly cancer patients.

\section{Financial \& competing interests disclosure}

The authors have no relevant affiliations or financial involvement with any organization or entity with a financial interest in or financial conflict with the subject matter or materials discussed in the manuscript. This includes employment, consultancies, honoraria, stock ownership or options, expert testimony, grants or patents received or pending, or royalties.

No writing assistance was utilized in the production of this manuscript.

\section{References}

1. Pasetto LM, Falci C, Compostella A, Sinigaglia G, Rossi E, Monfardini S. Quality of life in elderly cancer patients. Eur. J. Cancer 43(10), 1508-1513 (2007).

2. Monfardini S, Falci C, Brunello A, Lonardi S, Basso U. Cancer in the elderly. Intern. Emerg. Med. 6(Suppl. 1), 115-118 (2011).

3. Lichtman SM, Wildiers H, Chatelut E et al. International Society of Geriatric Oncology Chemotherapy Taskforce: evaluation of chemotherapy in older patients - an analysis of the medical literature. J. Clin. Oncol. 25(14), 1832-1843 (2007).
4. Wasil T, Lichtman SM. Treatment of elderly cancer patients with chemotherapy. Cancer Invest. 23(6), 537-547 (2005).

5. Esposito C, Plati A, Mazzullo T et al. Renal function and functional reserve in healthy elderly individuals. J. Nephrol. 20(5), 617-625 (2007)

6. Meneilly GS, Tessier D. Diabetes in elderly adults. J. Gerontol. A Biol. Sci. Med. Sci. 56(1), M5-M13 (2001).

7. Falci C, Basso U, Crivellari D et al. Advanced age, vulnerability/frailty and presence of comorbidities are associated with a delayed diagnosis made more by self examination than screening mammography in older breast cancer women: results of a prospective observational trial in 5 Italian centers. EJC Suppl. 7(2), 217 (2009).

8. Biganzoli L. Wildiers H, Oakman C et al. Management of elderly patients with breast cancer: updated recommendations of the International Society of Geriatric Oncology (SIOG) and European Society of Breast Cancer Specialists (EUSOMA). Lancet Oncol. 13(4), e148-e160 (2012). 